\title{
Practice guidelines for the treatment of hepatitis C: Recommendations from an AISF/SIMIT/SIMAST Expert Opinion Meeting
}

\section{A R T I C L E I N F O}

\section{Article history:}

Received 14 July 2009

Accepted 5 August 2009

Available online 11 September 2009

\section{Keywords:}

Antiviral therapy

Acute hepatitis

Chronic hepatitis

Cirrhosis

Elderly patients

HBV

$\mathrm{HCV}$

HDV

HIV

Liver transplantation

\begin{abstract}
A B S T R A C T
It is increasingly clear that a tailored therapeutic approach to patients with hepatitis $C$ virus infection is needed. Success rates in difficult to treat and low-responsive hepatitis $C$ virus patients are not completely satisfactory, and there is the need to optimise treatment duration and intensity in patients with the highest likelihood of response. In addition, the management of special patient categories originally excluded from phase III registration trials needs to be critically re-evaluated. This article reports the recommendations for the treatment of hepatitis $C$ virus infection on an individual basis, drafted by experts of three scientific societies.
\end{abstract}

\section{Introduction}

Hepatitis C virus (HCV) infection is a major health problem in Italy. Current estimates indicate that the number of chronically viremic HCV carriers exceeds 1.5 million (3\% of the population), with most of the infected subjects being older than 50 years. HCV infection is the main cause of cirrhosis and hepatocellular carcinoma in Italy and the main indication for liver transplantation.

National recommendations for the treatment of HCV have not been updated since 2003, when regimens based on the combination of pegylated interferons and ribavirin were adopted. However, treatment responses have varied widely in real-life practice, and it is increasingly clear that a tailored therapeutic approach to specific clinical situations is needed. In particular, success rates in difficult to treat and low-responsive HCV patients are still unsatisfactory, while treatment duration and intensity in patients with the highest likelihood of response must be optimised. Furthermore, the management of special patient categories

\footnotetext{
论 Position Paper on behalf of the following Scientific Societies: (1) Italian Association for the Study of the Liver (Associazione Italiana per lo Studio del Fegato: AISF); (2) Italian Society of Infectious, Tropical Diseases (Società Italiana per lo Studio delle Malattie Infettive e Tropicali: SIMIT); (3) Italian Society for the Study of Sexually Transmitted Diseases (Società Italiana per lo Studio delle Malattie Sessualmente Trasmissibili: SIMAST).
}

originally excluded from phase III registration trials needs to be evaluated.

These considerations prompted the organisation of an Expert Opinion Meeting with the aim of fine-tuning recommendations for the treatment of HCV infection on an individual basis. This was held in Naples in June 2008 and was endorsed by:

- The Italian Association for the Study of the Liver (Associazione Italiana per lo Studio del Fegato: AISF).

- The Italian Society of Infectious and Tropical Diseases (Società Italiana per lo Studio delle Malattie Infettive e Tropicali: SIMIT).

- The Italian Society for the Study of Sexually Transmitted Diseases (Società Italiana per lo Studio delle Malattie Sessualmente Trasmissibili: SIMAST).

This report summarises the proceedings of the meeting and its shared conclusions on orientations. Essential references are given at the end of document. Manuscripts based on the original presentations of each topic are available as supplementary materials at www.webaisf.org.

\section{Methods}

Primary objective of this document is to provide clinical practice guidelines defining the best management of individual categories of HCV patients, including those originally excluded from phase III 
registration trials and thus not covered by the current guidelines, since the lack of published data concerning some particular aspects of HCV therapy has led to heterogeneous indications and treatment modalities.

The format of recommendations through practice guidelines was chosen because the main objective was to offer clinically oriented readers practical suggestions for managing "difficult" HCV patients. To this aim, a Promoting Committee of AISF, SIMIT and SIMAST members identified multidisciplinary panels of 4-6 experts on nine controversial aspects of HCV infection (acute hepatitis, short or extended treatment, non-responders and relapsers, cirrhosis, liver transplantation, $\mathrm{HCV} / \mathrm{HIV}$ co-infection, $\mathrm{HCV} / \mathrm{HBV} \pm \mathrm{HDV}$ co-infection, elderly patients, and subjects with normal ALT). Each group analysed the literature and within 3 months presented a first draft of recommendations for review and suggestions to an external panel of clinicians acting as referees. A redrafted document was then discussed again with the referees during a public debate, after which the clinical practice guidelines were prepared.

The recommendations were drawn up using the method developed by Centro per la Valutazione della Efficacia della Assistenza Sanitaria (CeVEAS) di Modena (http://www.pnlg.it/cms/ files/Manuale_PNLG_0.pdf). The quality of the evidence backing any statement was scored as:

- Type (I): evidence based on more than one randomised controlled trial (RCT) or systematic review of RCT.

- Type (II): evidence based on a single randomised trial of adequate study design.

- Type (III): evidence based on non-randomised case-control cohort studies or systematic reviews of case-control cohort studies.

- Type (IV): evidence based on retrospective case-control studies or metanalysis of retrospective case-control studies.

- Type (V): evidence based on case series without control group.

- Type (VI): evidence based on expert opinions as indicated in guidelines documents or consensus conferences, or based on the opinion of the members of the working group responsible for the present document.

The grade (i.e. strength) of recommendation was based on the quality of evidence:

- Grade (A): the procedure or intervention is strongly recommended. Indicates a recommendation based on good quality evidence, not necessarily of type I or II.

- Grade (B): some uncertainties exist that the procedure or intervention should be always recommended; however, its application should be carefully considered.

- Grade (C): substantial uncertainties exist in favour against the procedure or intervention.

- Grade (D): the procedure or intervention is not recommended.

- Grade (E): the procedure or intervention is strongly discouraged.

\subsection{Treatment of acute hepatitis C (Group coordinated by Teresa Santantonio)}

\subsubsection{Background}

In Italy, HCV infection is responsible for about $10 \%$ of cases of acute viral hepatitis and the incidence of acute hepatitis C (AHC) is now about $1 / 100,000$ subjects per year. The current major risk factors for HCV transmission are intravenous drug use and viral exposure during medical procedures.

The gold standard for a definite diagnosis of AHC is the documented HCV-RNA or anti-HCV seroconversion (negative test within the previous 6 months). In the absence of documented serocon- version, diagnosis should be based on the presence of at least two of the following criteria: (a) ALT levels $>10$ times the upper normal limit; (b) known/suspected exposure to HCV within the previous 6 months; (c) exclusion of all other causes of acute liver damage.

Most AHC patients are asymptomatic with a generally mild clinical course. However, it has a high rate of chronicity (50-85\%, with spontaneous virus clearance occurring in only about $30 \%$ of patients. In self-limiting AHC patients, HCV generally clears during the first 3 months.

Most studies have evaluated the efficacy of conventional interferon (IFN) monotherapy and, recently, that of pegylated interferons (Peg-IFNs), although the latter are not licensed for AHC use. These studies showed that IFN monotherapy can reduce the risk of chronicity as it leads to higher rates of disease resolution and viral clearance than those observed in untreated patients. Using IFN monotherapy to treat $\mathrm{AHC}$ is therefore recommended by national and international guidelines, but it is still not standardised because of the difficulty in organising large-scale clinical trials to define how and when to treat.

Unlike chronic hepatitis $C$, the response of AHC to IFN therapy seems unaffected by viral genotype. IFN monotherapy is well tolerated even in patients with high ALT levels and jaundice, and reported side effects are similar to those in patients with chronic hepatitis $C$. Most studies have been conducted using IFN alpha or beta at a dose of 3-6 MU 3 times a week for 4-24 weeks, and a meta-analysis by Licata et al. definitely demonstrated that IFN monotherapy significantly increased the probability of a sustained viral response (SVR) in comparison with no treatment (risk difference $49 \%$ ). Moreover, the SVR rate is greater at higher weekly doses, thus indicating the need for higher IFN doses during the first month of therapy. In addition, the meta-analysis showed that delaying therapy up to 60 days after onset did not reduce the probability of a favourable response to standard IFN monotherapy.

More recently, other studies have investigated the efficacy of pegylated IFN (Peg-IFN) monotherapy using different strategies regarding treatment initiation, dose and duration. Published data indicate that Peg-IFN monotherapy leads to high SVR rates similar to those with conventional IFN plus the additional patient benefit of the administration of a single weekly dose. Response to treatment administered 8-12 weeks after clinical onset was similar to that obtained with earlier administration. The treatment duration can be reduced for patients with rapid virological response. Combination therapy with Ribavirin (RBV) seems unnecessary for AHC treatment, as the addition of RBV does not increase response in comparison with standard or Peg-IFN monotherapy.

\subsubsection{Statements}

1. A definite diagnosis of AHC should be based on the presence of HCV-RNA in the blood of a previously HCV negative patient or seroconversion (EIA) of a negative anti-HCV test (in the previous 6 months) into positive. In the absence of documented seroconversion, the diagnosis should be based on the presence of at least two of the following criteria: (a) ALT levels $>10$ times the upper normal limit; (b) known or suspected exposure to HCV within the previous 6 months; (c) the exclusion of all other causes of acute liver damage (A-III).

2. When no precise diagnosis of AHC can be made, a liver biopsy should be considered in order to exclude a reactivation of chronic hepatitis $\mathrm{C}(\mathrm{B}-\mathrm{VI})$.

3. An average of only $30 \%$ of $A H C$ patients experience spontaneous viral clearance, which primarily occurs during the 3 months following the clinical onset of the disease (B-III).

4. All patients with AHC should be considered for treatment in order to prevent progression to chronic hepatitis C (A-I). 
5. Delaying treatment for 8-12 weeks after disease onset allows the identification of subjects in whom the infection spontaneously resolves, thus avoiding unnecessary therapy (A-II).

6. AHC treatment is based on IFN monotherapy, which leads to high sustained virological response rates (>90\%) (A-I). IFN/ribavirin combination therapy is not more effective than IFN monotherapy in AHC patients, although it is more effective in chronic hepatitis $C$ patients (A-II).

7. Pegylated interferon monotherapy may be the best therapeutic option as its efficacy is comparable with that of standard IFN monotherapy (A-III).

8. An induction regimen with daily doses of conventional IFN is associated with higher SVR rates (A-III). When using Peg-IFN alpha-2b, the recommended $1.5 \mu \mathrm{g} / \mathrm{kg} /$ week dose should be administered (A-III).

9. The optimal duration of IFN monotherapy seems to be 24 weeks, but a shorter course may be as effective (B-III). A 12-week course of Peg-IFN is effective in patients with undetectable HCV-RNA levels by week 4 (B-III).

10. The response of AHC to IFN therapy does not seem to be affected by viral genotype or pre-treatment viral load (B-III).

11. There is no indication for administering IFN as post-exposure prophylaxis or for the treatment of subjects with primary HCV infection without and clinical signs of disease (B-VI).

\subsection{Individualised therapy for chronic hepatitis C (Group coordinated by Alfredo Alberti)}

\subsubsection{Background}

The current standard of care (SOC) for the treatment of chronic hepatitis C and HCV-related compensated cirrhosis is the combination of a pegylated IFN (Peg-IFN alpha2a or Peg-IFN alpha2b) and ribavirin. On the basis of the evidence-based data produced by randomised clinical trials, current treatment guidelines recommend administering this therapy for 48 weeks to patients infected by HCV-1 (HCV-1a or HCV-1b) or HCV-4, and for 24 weeks to those infected by HCV-2 or HCV-3.

The same guidelines recommend stopping antiviral therapy after 12 weeks in HCV-1 or HCV-4 infected patients if their HCVRNA levels have not decreased by at least $2 \log 10$ in comparison with baseline on the basis of solid evidence showing that such patients have little or no likelihood of achieving a sustained viral response (SVR) when treated for $48-52$ weeks. No similar recommendations have been proposed for patients with HCV-2 and $\mathrm{HCV}-3$ infection.

Using the current SOC regimens and adequate doses of Peg-IFN and ribavirin, SVR rates are as high as 70-85\% in patients with HCV2 or HCV-3, intermediate in those with HCV-4, and lower $(<50 \%)$ in patients with $\mathrm{HCV}-1$. Recent data indicate that patients with HCV-2 and HCV-3, traditionally considered an "easy-to-treat" category, have different SVR rates and should be considered separately, although doses and treatment duration have not yet been identified for the two genotypes.

A number of virus- and host-related variables have been identified as influencing SVR rates in HCV-infected subjects. In addition to the HCV genotype, baseline viremia levels are certainly important in the case of patients infected by HCV-1 and HCV-3, although less so for those infected by HCV-2. The host-related factors include age, alcohol intake, the stage of liver disease, obesity and, obviously, treatment adherence. A number of co-morbidities also affect SVR rates, above all HBV and HIV co-infections and the metabolic syndrome with insulin resistance, but also any associated condition that reduces adherence to adequate PEG-IFN or ribavirin dosing.

There is good reason to believe that the current SOC of HCV therapy, with its two fixed 48 - or 24 -week regimens based on the infecting HCV genotype, is not ideal and may lead to the over-treatment of the most easily treated patients and the undertreatment of those are more difficult to treat. Grouping HCV-1 and HCV-4 cases as difficult to treat and always requiring 48 weeks' treatment, and HCV-2 and HCV-3 cases as easy to treat and best suited to a 24-week regimen, is an oversimplification that may facilitate a pragmatic approach to HCV therapy but is rather weak on biological and evidence-based grounds. This situation has led to growing interest in the possibility of further individualising treatment duration in patients with chronic hepatitis $C$ by means of response-guided therapy (RGT), an approach supported by the fact the early kinetics of the virological response (i.e. the rapidity and degree of HCV-RNA decay in serum during the first 4-12 weeks of treatment) allows the categorisation of treated patients on the basis of their "susceptibility" to HCV eradication and the definition of more individualised treatment durations regardless of the infecting HCV genotype.

The principles of individualised treatment approach are different for HCV-1 and HCV-2 patients.

2.2.1.1. HCV-1 patients. A number of studies have compared SVR rates in HCV-1 infected patients treated for 24 or 48 weeks. In a randomised study of Peg-IFN alpha2a in patients with HCV-1 in which the duration of treatment was based on a rapid viral response (RVR: negative HCV-RNA [ $<50 \mathrm{IU} / \mathrm{mL}]$ by week 4$)$, Ferenci et al. observed an intention to treat-SVR rate of $75 \%$. In an open, non-randomised cohort study of patients treated with Peg-IFN alpha2b for 24 weeks, Zeuzem et al. found that the SVR rate in those with HCV-1 and a low baseline viral load $(<600,000 \mathrm{IU} / \mathrm{mL})$ was only $50 \%$ as against to a historical control value of $71 \%$ with 48 weeks' therapy, but a subgroup analysis of the patients with an RVR indicated an SVR rate of $88 \%$ vs. $91 \%$. Despite the weak study design and the retrospective nature of the subgroup analysis, these results were deemed adequate to grant an indication for 24-week treatment in HCV-1 patients with a low baseline viral load and RVR.

Janssen et al. retrospectively analysed the results of Peg-IFN alpha2a registration trials, and found that patients with a rapid virological response (RVR) had similar SVR rates when treated for 24 or 48 weeks ( $88 \%$ and $91 \%$ ); an RVR was seen in $16 \%$ of the HCV1 patients but this was reduced in the presence of high baseline viremia, HCV-1a, and advanced liver fibrosis.

Mangia et al. compared the results of a randomised study of HCV-1 infected patients treated with Peg-IFN alpha2a or PegIFN alpha2b on a fixed 48-week schedule, and those observed in patients whose duration of therapy was based on the time to HCVRNA negativity. When patients with an RVR were considered, the SVR rate was $87.1 \%$ in those treated for 48 weeks and $77.2 \%$ in those treated for 24 weeks; this difference was not statistically significant mainly because of the limited number of patients. Relapse rates were higher in the patients with high baseline viremia levels $(>400,000 \mathrm{IU} / \mathrm{mL})$.

Other studies have compared treating HCV-1 patients for 48 and 72 weeks in an attempt to identify those who may benefit from a longer treatment duration than that foreseen in the current SOC. Berg et al. compared Peg-IFN alpha2a combined with a fixed $800 \mathrm{mg}$ dose of ribavirin and found no difference in SVR rates between 48 and 72 weeks; however, a retrospectives analysis showed that the SVR rates with both regimens were similar in patients with an RVR or a complete early virological response (cEVR: HCV-RNA positive at week 4 but negative at week 12), but significantly higher with 72 weeks (46\% vs. 33\%) in the patients with a partial EVR (pEVR: HCV-RNA positive at week 4 and 12 , but a $\geq 2 \log 10$ decrease from baseline at week 12). Similar findings were reported by SanchezTapias et al., who found that the SVR rates in HCV-1 patients with a pEVR were $16 \%$ when treated for 48 weeks and $44 \%$ when treated for 72 weeks, and these differences were also confirmed by a small 
study of Ferenci et al. in which the corresponding figures were 31\% and $77 \%$. All three studies found that 72 weeks' treatment offered no benefit over 48 weeks' treatment in terms of SVR rates in patients with a cEVR or without an EVR. They also confirmed the extremely low likelihood of an SVR in patients without an EVR regardless of the duration of treatment.

2.2.1.2. HCV-2 and HCV-3 patients. A number of studies have investigated whether patients with $\mathrm{HCV}-2$ or $\mathrm{HCV}-3$ (or a subgroup of them) can be treated with Peg-IFN and ribavirin for less than the current SOC of 24 weeks without reducing SVR rates. Most of them pooled HCV-2 and HCV-3 patients, although they should be analysed separately because of the growing evidence that they respond differently to Peg-IFN plus ribavirin.

The studies have assessed shortening the treatment schedule to 12,14 or 16 weeks, but their findings are limited by the heterogeneity of their designs, the use of different cut-off values to define virological response at different time points, the use of different doses of ribavirin in combination with different types of Peg-IFN, and their small patient populations (particularly when it is wanted to consider HCV-2 and HCV-3 separately).

The studies assessing the possibility of shortening treatment in $\mathrm{HCV}-2$ patients had designs based on allocation or randomisation by RVR. Mangia et al. found no difference in SVR when the patients with an RVR were treated for 12 or 24 weeks (87\% vs. $89 \%$ ), and similar results were obtained by von Wagner et al. (SVR: $95 \%$ after both 16 and 24 weeks' therapy) and Dalgard et al. (93\% after 14 weeks vs. $97 \%$ after 24 weeks).

On the other hand, the subgroup analysis of HCV patients developing an RVR in the large "ACCELERATE" clinical trial showed that the SVR rate was lower after 16 weeks that after 24 weeks of therapy ( $80 \%$ vs. 91\%), although this study did not allocate the patients to short or standard treatment on the basis of RVR.

All of these studies confirm that the presence of an RVR is associated with high SVR rates, whereas its absence reduces the likelihood of an SVR after 24 weeks of treatment to $<50 \%$. The same studies also analysed patients with HCV-3, and all of them found that an RVR was highly predictive of SVR. However, some of them found that the RVR and SVR rates in the HCV-3 patients were different from and generally about 10\% lower than those observed in the HCV-2 patients, thus confirming that the two genotypes respond differently to treatment with Peg-IFN plus ribavirin. In the study by Mangia et al., $77 \%$ of the HCV-3 patients with an RVR achieved an SVR when treated for 12 weeks, as against $100 \%$ of those treated for 24 weeks. Response rates after 24 weeks were better than those after 14 weeks $(92 \%$ vs. 84\%) in the study by Dalgard et al., whereas von Wagner et al. Found no difference between 16 weeks and 24 weeks of treatment (76\% vs. 75\%) and the same was true of the "ACCELERATE" study ( $84 \%$ vs. $89 \%$ ). These heterogeneous results mainly reflect the small size of the study populations, the different study designs, the different types of Peg-IFN, and the different ribavirin doses. All of the studies indicated that an RVR is associated with high SVR rates, whereas the absence of an RVR reduces the probability of an SVR to $<50 \%$. The RVR and SVR rates were significantly lower in the patients with high baseline viremia levels and in those with advanced fibrosis or cirrhosis.

\subsubsection{Statements}

2.2.2.1. $\mathrm{HCV}-1$.

1. In patients with HCV-1, an RVR is an important predictor of a SVR (A-I).

2. In patients with HCV-1, the absence of an EVR is an important predictor of non-response, with a very high negative predictive value. Therapy should therefore be stopped in the absence of an EVR (at least a 2 log reduction in HCV-RNA levels in comparison with baseline) (A-I).

3. The duration of Peg-IFN and ribavirin treatment can be reduced to 24 weeks in some HCV-1 patients (AII). To do this safely without compromising SVR rates, all of the following conditions should be fulfilled:

- baseline HCV-RNA $<600,000 \mathrm{IU} / \mathrm{mL}$

- an RVR after 4 weeks of treatment

- optimal adherence to the Peg-IFN and ribavirin doses

- the absence of major co-factors known to reduce response (HBV or HIV co-infection, obesity and metabolic syndrome, advanced fibrosis or cirrhosis)

4. Patients with HCV-1 and a pEVR should be treated for 72 weeks in order to maximise the chance of an SVR. The decision to prolong therapy in these patients should take individual side effects, the quality of life and the patient's motivation into account (A-I).

5. Patients with HCV-1 and without an RVR but with a cEVR should be treated for 48 weeks (A-I).

6. All patients should received standard doses of Peg-IFN alpha2a or alpha2b and weight-based doses of ribavirin as SOC (A-II).

7. Using higher Peg-IFN and/or ribavirin doses to improve SVR rates in patients with HCV-1 is still experimental and cannot be recommended in clinical practice (A-II).

\subsubsection{2. $\mathrm{HCV}-2$ and $\mathrm{HCV}-3$.}

1. The SOC for the treatment of patients with HCV-2 and HCV-3 is the combination of standard doses of Peg-IFN alpha 2a or alpha $2 \mathrm{~b}$ and weight-based doses of ribavirin given for 24 weeks (A-I).

2. Patients with HCV-2 or HCV-3 and an RVR have a very high chance of achieving an SVR (A-I).

3. The duration of treatment can be shortened to 12-16 weeks in a subgroup of patients with HCV-2 who have developed RVR. To do this safely without compromising SVR rates, all the following conditions should be fulfilled: the presence of an RVR; the absence of advanced fibrosis, cirrhosis or any other cofactor/ comorbidity known to reduce the efficacy of antiviral therapy (including HIV, HBV co-infection, obesity, metabolic syndrome); and adequate adherence to Peg-IFN and ribavirin. Shortening therapy should be considered particularly in the presence of side effects that may be expected to worsen with continued treatment (A-II).

4. The duration of treatment can be shortened to 12-16 weeks in a subgroup of patients with HCV-3 who have developed an RVR. To do this safely without compromising SVR rates, all of the following conditions should be fulfilled: low baseline viremia levels $(<600,000 \mathrm{IU} / \mathrm{mL})$; the presence of an RVR; the absence of advanced fibrosis, cirrhosis, severe steatosis, or any other co-factor/co-morbidity known to reduce the efficacy of antiviral therapy (including HIV, HBV co-infection, obesity, metabolic syndrome); and adequate adherence to Peg-IFN and ribavirin. Shortening therapy should be considered particularly in the presence of side effects that may be expected to worsen with continued treatment (A-II).

5. Although the SVR rates obtained by means of 24 weeks' treatment in HCV-2 and HCV-3 patients without an RVR are comparatively low, none of the available data supports the use of longer treatment duration (CII).

6. The rates of response to retreatment in HCV-2 and HCV-3 patients with an EVR who relapse after a short treatment course are currently unknown. None of the available data supports the use of longer treatment duration (C-II). 


\subsection{Retreatment of non-responders and relapsers (Group coordinated by Antonio Craxi)}

\subsubsection{Background}

Since the early 2000 s, at least $50 \%$ of chronic hepatitis $C$ patients have failed to respond to treatment with standard interferon alpha (IFN alpha) and ribavirin combination therapy and there is still a large cohort of non-responders (i.e. subjects with detectable serum HCV-RNA 3 or 6 months after the start of therapy). The clinical course of the disease seems to be worse in these patients, leading to an accelerated progression towards end-stage liver disease and the development of hepatocellular carcinoma. An effective retreatment regimen is therefore a major goal in their long-term management.

A number of studies of retreatment with Peg-IFN plus ribavirin in patients failing to respond to the combination of standard or pegylated IFN and ribavirin have been published. However their results are inconclusive or conflicting because of the relatively small study populations, and difficult to generalise because of differences in patient characteristics, study designs including relapsers and non-responders, and different IFN and ribavirin doses in the first course and retreatment regimens.

To evaluate better the results of these studies, a meta-analysis was made in accordance with the QUOROM statement by retrieving trials from the Cochrane Controlled Trials Register, the Cochrane Library, MEDLINE and ENBASE using the following medical subject headings: chronic hepatitis $\mathrm{C}$, non-responders, interferon and ribavirin, pegylated interferon, retreatment, clinical trial. The search was carried out in May 2008, without a lower date limit on the search results. After reviewing the titles and abstracts, 20 articles were considered to fulfil the inclusion criteria and were selected for review. Twelve, which accounted for 1571 patients (30.9\%), were reported as full papers, and eight studies, which accounted for 3508 patients (69.1\%), were abstracts. Three of the full-length papers did not report the number of participating centres; the others all involved between 2 and 23 centres. Eighteen were prospective cohort studies; two were randomised controlled trial (RCTs).

2.3.1.1. Sustained virological response rate (SVR). Treatment regimens varied widely in terms of the type of Peg-IFN (alpha-2a or alpha-2b); the dose of Peg-IFN alpha-2b (50-300 $\mu \mathrm{g} /$ week); and the dose of ribavirin (800-1400 mg/day). The duration of retreatment was 48 weeks in all but one trial.

The 20 studies involved a total of 5079 patients, and the estimated pooled SVR rate was $16 \%$ (95\% CI 6-33\%). The magnitude of the treatment effect was remarkably heterogeneous (chi-squared test for heterogeneity 159.5 with $19 \mathrm{DF} ; p<0.0001$ ). The proportion of patients who achieved an SVR differed widely from 6\% [88] to $32 \%$.

Only six trials provided data concerning SVR rates by genotype (genotype $1 v s$. genotype non-1). The estimated pooled SVR rate in the subgroups of these six studies was $15.6 \%$ for genotype 1 (95\% CI 12.4-19.4\%) and 33.9\% for genotype non-1 (95\% CI 25.8-43.1\%) $(p=0.0001)$.

\subsubsection{Statements}

Concerning retreatment with a 48-week course of pegylated IFN plus ribavirin in non-responders to standard or pegylated IFN and ribavirin combination therapy, the available evidence is sufficient to conclude that:

1. The low levels of overall efficacy (16\% SVR) and tolerability, and the inconsistent SVR rates argue against the indiscriminate retreatment of all non-responders to combination therapy (B-III)

2. Restricting retreatment to patients infected with HCV genotype 2 or 3 optimises the potential benefit (34\% SVR) (A-III)
3 . The use of a stopping rule after $12-24$ weeks of retreatment avoids unnecessarily long and unsuccessful treatments (A-III)

4. Given the low probability of clinical benefit, the decision to retreat subjects infected with HCV genotype 1 should be assessed on an individual basis (C-III)

\subsection{Antiviral treatment in HCV cirrhosis (Group coordinated by Savino Bruno)}

\subsubsection{Background}

Cirrhotic patients have a significant risk of developing hepatic decompensation within a decade, and a $1-4 \%$ yearly risk of developing hepatocellular carcinoma (HCC) within 5 years after diagnosis. HCV cirrhosis is now the most common reason for liver transplantation. Recent studies show that achieving a sustained virological response (SVR) obtains a sharp reduction in liver-related mortality and morbidity, including HCC in patients with compensated HCV cirrhosis.

In the landmark phase III trials assessing combined pegylated interferon (Peg-IFN) plus ribavirin (RBV), 15-30\% of the patients showed signs of severe liver disease, and SVR rates were calculated by pooling all of those with bridging fibrosis (Knodell score F3) and complete cirrhosis (Knodell score F4), thus including patients at very different stages of liver disease ranging from marginal bridging fibrosis to compensated cirrhosis. Since the latter were no more than $6 \%$ of the patients distributed in the different arms of the studies, no reliable conclusions could be reached concerning the safety and efficacy of Peg-IFN alpha2b or Peg-IFN alpha2a plus RBV in patients with HCV-induced cirrhosis. Moreover, the SVR rates according with "easy-to-treat" (2 and 3) and/or "difficultto-manage" genotypes ( $1 \mathrm{a}, 1 \mathrm{~b}$ and 4 ) in this subset of patients are poorly investigated. Finally, the reliability of baseline and ontreatment predictors of response together with the efficacy of the SOC schedule of treatment currently used in subjects with mild to moderate fibrosis needs to be assessed in cirrhotics by dedicated studies.

Due to the heterogeneity in clinical stages of cirrhosis, even within the same Child-Pugh class, the following classification of patients with HCV cirrhosis was used to assess the results:

- Patients with "histologically proven" cirrhosis without oesophageal varices (Child class A5 to 6) identified by stages 5 and 6 of Ishak's score, and stage 4 of the Metavir and Knodell scores

- Patients with "compensated" cirrhosis with or without oesophageal varices (including Child class B7) identified by a clinical or histological diagnosis of cirrhosis; bilirubin and albumin levels of $<2 \mathrm{mg} / \mathrm{dL}$ and $>2.8 \mathrm{~g} / \mathrm{dL}$; a prothrombin time of >60\%; HVPG > $5 \mathrm{mmHg}$; oesophageal varices and/or platelet levels of $>100,000 / \mathrm{dL}$; and a bipolar spleen diameter of $<13 \mathrm{~cm}$ without any previous episode of decompensation or evidence of HCC

- Patients with "decompensated" cirrhosis: (Child class B8 or more) defined by any evidence of previous decompensation (ascites, oesophageal bleeding, portal encephalopathy, jaundice)

The achievement of an SVR, defined as undetectable RNA levels $(<50 \mathrm{IU} / \mathrm{mL}) 6$ months after the end of antiviral therapy, is a reliable prognostic marker in patients with HCV cirrhosis regardless of the stage. SVR is associated with a reduction in: (1) decompensation, occurrence of HCC, and mortality in patients with histologically proven cirrhosis without oesophageal varices; (2) event rates in patients with oesophageal varices; and (3) mortality in patients at a decompensated stage of the disease. 
The following statements are based on a systematic review of the medical literature, including articles published in peer reviewed journals between 2001 and 2008 and abstracts selected for the period from the 2005 AASLD meeting to the 2008 EASL meeting (DDW, AASLD, EASL, APASL, AISF). Eleven papers and eight abstracts were considered in the final analysis.

\subsubsection{Statements}

1. For patients with "histologically proven" cirrhosis without oesophageal varices (Child class A5 to 6):

- The SVR rate in naïve patients ranges from $25 \%$ to $76 \%$ (A-I)

- The SVR rate in previous non-responders is approximately $10 \%$ (A-II)

- Achieving an SVR is more frequent in patients with genotypes 2 or $3(76-78 \%$ and $17-55 \%)$ than in patients with genotype $1(25 \%$, range $24-27 \%$ ) (A-II)

- A rapid virological response (RVR), early virological response (EVR) and genotype are the main predictors of an SVR (A-II)

- The rate of treatment withdrawal is higher than in patients without cirrhosis (A-I)

- Bone marrow toxicity (but not other toxicities) is more frequent than in patients without cirrhosis (A-I)

- The achievement of an SVR is associated with a decrease in the decompensation rate, liver-related deaths, and the occurrence of HCC (A-III)

- Three-years long "maintenance" therapy with Peg-IFN alpha 2a/b monotherapy did not reduce the rate of decompensation, HCC and mortality (A-II)

- Given the lack of studies comparing a standard duration (48 weeks) with short duration treatment ( 24 weeks) in patients with "easy-to-treat" genotypes (2 and 3), and extended treatment (72 weeks) in patients with "difficult-to-treat" genotypes (1 and 4), no indication can be given concerning individual genotype subpopulations of cirrhotic patients

- Clinical gain is extremely relevant as an SVR is associated with a significantly better outcome (A-III)

- Naïve patients should be given antiviral therapy because of the high rate of SVRs (A-I)

- Due to the low rate of SVR, it is nowadays not advisable to re-treat non-responder patients (A-II).

- Non-responder patients should not be treated with 3-years long "maintenance" therapy (A-II)

2. For patients with "compensated" cirrhosis with or without oesophageal varices (including Child class B7):

- The SVR rate is approximately $20 \%$ (A-III)

- The achievement of an SVR is more frequent in patients with genotypes 2 or $3(50 \%$ and $60 \%$ ) than in patients with genotype 1 (10-18\%) (A-III)

- Baseline predictors of an SVR are genotype and albumin levels; on-treatment predictors of are an RVR and EVR (A-III)

- The rate of treatment withdrawal is similar to those observed in patients with "histologically proven" cirrhosis without oesophageal varices (A-III)

- The rate of bone marrow toxicity is similar to that of patients with "histologically proven" cirrhosis without oesophageal varices (AIII)

- The achievement of an SVR is associated with a significant decrease in the rates of decompensation and HCC (A-III)

- There are no data concerning the optimal duration of therapy as all of the patients were treated for 48 weeks

- Patients can be treated mainly if they are infected by an "easyto-treat" genotype (A-III)
- Clinical gain is relevant as an SVR is associated with a significantly better outcome (A-III)

3. For patients with decompensated cirrhosis:

- The SVR rate is approximately $20 \%$ (B-III)

- The achievement of an SVR is more frequent in patients with genotypes 2 or 3 (43\% cumulatively) than in patients with genotype 1 and 4 (approximately 7\%) (B-III)

- The rate of severe side effects (mainly infections) is significantly higher than in untreated patients (A-III)

- The achievement of an SVR may be associated with improved decompensation in comparison with untreated patients or nonresponders, thus delaying the need for a liver transplant. Further studies are needed to confirm these data (C-III)

- There are no data concerning the optimal duration of therapy as all of the patients were treated for 6 months

- These patients should generally not be treated outside trials in the transplant setting.

4. For patients with cirrhosis and HIV co-infection:

- Treatment can be successful (A-I)

- The management of anti-HCV therapy is difficult in this setting (A-III)

- Anti-HIV treatment can delay the onset of decompensation and improve the response to anti-HCV therapy (A-I)

- Nucleosides analogues must be used cautiously as in the case of patients without cirrhosis (B-I)

- Disease progression can be slowed by achieving an SVR (A-III).

\subsection{Treatment of HCV reinfection after liver transplantation (Group coordinated by Stefano Fagiuoli and Paolo Grossi)}

\subsubsection{Background}

HCV-related end-stage liver disease (ESLD) is the main indication for liver transplantation, accounting for $30-40 \%$ of transplants. Post-transplantation HCV recurrence is virtually universal in the carriers of active infection. Histological recurrent disease can be documented in $80-90 \%$ of recipients 5 years after transplantation, and within the same time frame, evolution towards cirrhosis can be expected in $25-30 \%$ of recipients. More than $35 \%$ of graft losses in HCV-related ESLD are due to disease recurrence. After the development of cirrhosis, the mean time to first decompensation is dramatically shorter than that observed during the natural course of the disease and explains the significantly higher mortality in HCV-related liver transplantations in comparison with non-HCV indications.

Antiviral treatment of the recurrence of HCV after liver transplantation should be proposed only by a hepatologist who is expert in both chronic HCV hepatitis and liver transplantation, in agreement with the referring Liver Transplant Centre. A selection of the most important statements, based on a review of the medical literature is reported below. The complete list of statements is available online at www.webaisf.org.

\subsubsection{Statements}

1. The recurrence of HCV-related disease after liver transplantation significantly reduces graft and patient survival (A-III)

2. Two treatment approaches can be considered:

(a) Pre-emptive therapy: "very early" treatment of graft reinfection (within 4-6 weeks of transplantation) before the virological, clinical or histological features of HCV develop. This approach is not advisable because of the risk of acute and chronic rejection and the poor tolerance of patients 
Moreover, pre-emptive therapy exposes a large number of patients who will not develop liver disease to unnecessary treatment (A-III).

(b) Antiviral treatment of HCV-related liver disease: treatment is proposed when fibrosis is $\geq F 2$ ) (B-III).

3. The inclusion criteria for antiviral therapy are the following: HCV-RNA positivity; histology consistent with recurrent HCV and fibrosis stage $\geq F 2$; absence of rejection, biliary obstruction and vascular damage (B-III).

4. Combination therapy is currently recommended and the most widely used is pegylated IFN plus ribavirin (A-III).

5. There is no evidence of any difference in the efficacy or tolerability of Peg-IFN alpha2a and Peg-IFN alpha2b after liver transplantation (A-III), and the suggested dose is

- Peg-IFN alfa2a: $180 \mu \mathrm{g} /$ week s.c.; Peg-IFNalfa2b: $1.5 \mu \mathrm{g} /$ week s.c.

- Ribavirin 1000-1200 mg/day p.o.

6. No data support the efficacy of dose reductions or escalations, therefore they are not recommended (D-VI).

7. The most widely used antiviral treatment schedule is 48 weeks (B-III)

8. The absence of an early virological response (EVR, defined as a reduction in HCV-RNA of $<2 \log$ after 12 weeks) significantly predicts non-response to treatment (NPV $=95-100 \%$; $P P V=50-60 \%)$. For this reason the therapy should be stopped, although there are still insufficient data to define this approach partially because of the lack of a precise definition of the primary objective of antiviral treatment after liver transplantation (C-II).

9. The most common side effects of antiviral therapy are anaemia, leucopoenia and thrombocytopenia, which have been reported in more than $50 \%$ of treated patients (A-II).

10. The use of growth factors should make it possible to prolong antiviral treatment, thus limiting the need for a dose reduction and ensuring a better quality of life (A-II).

11. No cut-off in $\mathrm{Hb}$ or neutrophil values have been established for the use of growth factors. In usual practice, erythropoietin is administered when $\mathrm{Hb}$ is $<10 \mathrm{~g} / \mathrm{dL}$ and/or the reduction in $\mathrm{Hb}$ is $\geq 2 \mathrm{~g} / \mathrm{dL}$, and growth factors are used when neutrophils are $<500 / \mathrm{mm}^{3}$ (C-III).

12. Usual doses of growth factors are the following: for erythropoietin 40,000 U/week s.c.; for G-CSF, $300 \mu \mathrm{g} \times 1-2 /$ week s.c., but none of these schedules has been validated in the liver transplant setting $(\mathrm{C}-\mathrm{VI})$.

The statements regarding the antiviral treatment of HIV/HCV co-infected patients after liver transplantation are available online at www.webaisf.org.

\subsection{Treatment of HIV/HCV co-infection (Group coordinated by Raffaele Bruno)}

\subsubsection{Background}

Fibrosis staging is important for making therapeutic decisions in co-infected patients, but a liver biopsy is not mandatory for decisions concerning the treatment of chronic HCV infection, also due to the unpredictable, usually fast evolution of fibrosis in these patients.

Current SOC therapy is particularly recommended in patients with a high likelihood of achieving a sustained virological response (SVR): i.e. those infected by genotypes 2 or 3 , and those infected with genotype 1 if the viral load is low $(<400,000-600,000 \mathrm{IU} / \mathrm{mL})$; in the case that a liver biopsy or non-invasive tests of hepatic fibrosis (e.g. transient elastometry, FibroScan, Echosens, [Paris], France) have demonstrated lower grades of liver fibrosis (F0-F1), treatment can be deferred regardless of HCV genotype. It is especially important to assess the stage of liver disease in patients unlikely to achieve an SVR.

The standard weekly doses of Peg-IFN alpha2a and Peg-IFN alpha $2 \mathrm{~b}$ are respectively $180 \mathrm{mg} / \mathrm{kg}$ and $1.5 \mathrm{mg} / \mathrm{kg}$. A weightadjusted ribavirin (RBV) daily dose of between $1000 \mathrm{mg}$ (weight $<75 \mathrm{~kg}$ ) and $1200 \mathrm{mg}$ (weight $>75 \mathrm{~kg}$ ), given in two administrations, is recommended for all genotypes If chronic hepatitis $C$ is detected early in the course of HIV infection (before the start of HAART), HCV treatment is advised but, if a co-infected patient is severely immunodeficient (a CD4+ cell count of less than 200 cells/mL), this should be improved by highly active antiretroviral therapy (HAART) before starting anti-HCV treatment. Patients with relative CD4 counts of $25 \%$ are more likely to achieve an SVR than those with lower percentages.

Rapid Virological Response (RVR) at week 4 is correlated with higher SVR rate. If no early virological response (a reduction of at least $2 \log _{10}$ in HCV-RNA in comparison with baseline) is achieved by week 12 , HCV treatment should be discontinued, as an SVR is unlikely.

During the course of PEG-IFN alpha plus RBV therapy, didanosine is contraindicated, and stavudine and zidovudine should be avoided if possible.

\subsubsection{Statements}

1. HCV treatment makes it possible to eradicate HCV, which is potentially advantageous for the subsequent management of patients with HIV. All such patients should therefore be considered for treatment when its benefits outweigh the risks (A-IV).

2. HCV therapy is particularly recommended in patients who are highly likely to achieve a sustained virological response (SVR): i.e. those with genotypes 2 or 3 , and those with genotype 1 provided that their viral load is low $(<400,000-600,000 \mathrm{IU} / \mathrm{mL})$ (A-I).

3. If a liver biopsy or non-invasive test demonstrates low grades of liver fibrosis (F0-F1), treatment can be deferred regardless of the HCV genotype (A-VI).

4. If chronic hepatitis $C$ is detected early in the course of HIV infection (before the start of HAART), HCV treatment is advisable (A-VI).

5. If a co-infected patient is severely immunodeficient (CD4 count $<200$ cells $/ \mathrm{mL}$ ), the CD4 count should be improved by HAART before starting anti-HCV treatment. Patients with relative CD4 counts of $25 \%$ are more likely to achieve an SVR than those with lower percentages (A-IV).

6. Combined Peg-IFN and weight-based ribavirin treatment is the SOC for HCV infection. The standard weekly doses of PegIFN alpha2a and Peg-IFN alpha2b are respectively $180 \mathrm{mg} / \mathrm{kg}$ and $1.5 \mathrm{mg} / \mathrm{kg}$. A weight-adjusted RBV daily dose of between $1000 \mathrm{mg}$ (weight $<75 \mathrm{~kg}$ ) and $1200 \mathrm{mg}$ (weight $>75 \mathrm{~kg}$ ), given in two administrations, is recommended for all genotypes. The recommended duration of treatment for all genotype is 48 weeks, except for genotype 1 patients not achieving an HCVRNA negative at week 4 and a drop more than two log of HCV-RNA at week 12 which should be treated for 72 weeks (A-I).

7. If no early virological response (a reduction of at least $2 \log _{10}$ in HCV-RNA in comparison with baseline) is achieved by week 12 , HCV treatment should be discontinued as an SVR is unlikely (A-I)

8. Patients with genotypes 2 or 3 , low viral loads $(<400,000 \mathrm{U} / \mathrm{mL})$ and mild fibrosis in whom HCV-RNA becomes undetectable in 4 weeks (a rapid virological response) may need only 24 weeks of therapy, but this can increase the relapse rate (A-III). 
9. $\mathrm{HIV} / \mathrm{HCV}$ co-infection may suggest starting antiretroviral therapy early (CD4 counts of $350-500$ cells $/ \mathrm{mm}^{3}$ ) because the therapy itself and higher $\mathrm{CD} 4$ counts may slow the progression of liver disease (B-III).

10. During Peg-IFN plus ribavirin therapy, didanosine is contraindicated, and stavudine and zidovudine should be avoided if possible (I-A).

11. In HIV/HCV co-infected patients, liver function tests should be performed 1 month after starting therapy, 3 and 6 months later, and then every 3 months $(\mathrm{A}-\mathrm{VI})$.

12. Antiretroviral therapy should be promptly withdrawn in the case of: (1) lactic acidosis; (2) hypersensitivity reactions; (3) liver function test results $>10$ times the upper normal limit or five times the values observed before therapy; or (4) jaundice or signs of liver decompensation (A-VI).

\subsection{Treatment of $H C V / H B V \pm H D V$ co-infection (Group coordinated by Giovanni Battista Gaeta)}

\subsubsection{Background}

2.7.1.1. $\mathrm{HCV} / \mathrm{HBV}$ coinfection. HBV coinfection has a prevalence of $1-2 \%$ among patients with HCV chronic infection. These patients are usually negative for HBeAg. A more consistent prevalence is found in high-risk groups (HIV coinfected individuals, IVDU).

In chronic $\mathrm{HCV} / \mathrm{HBV}$ coinfection, cross-sectional studies show a reciprocal ability of inhibition, usually with a prevalent negative influence of HCV on HBV. A prospective Italian study clarifies that the replicative status of one or both the viruses may vary over time in at least $30 \%$ of patients. This observation is of great relevance for defining the therapeutic approach in each patient. IFN-RBV combination treatment leads to a rate of sustained virological response analogous to that found in HCV monoinfected individuals. However, HCV suppression was associated HBV reactivation in some patients independently from the treatment received.

2.7.1.2. $H C V / H B V / H D V$ coinfection. Infection with $\mathrm{HCV}, \mathrm{HBV}$, and HDV can induce a severe disease with increased risk of hepatocellular carcinoma (III-A). The statements emphasise the need for antiviral therapy but at present few data, not conclusive, are available on treatment schedule and efficacy.

\subsubsection{Statements}

2.7.2.1. $\mathrm{HCV} / \mathrm{HBV}$ coinfection.

- Virological profiles of patients with chronic HCV/HBV coinfection must be defined before starting therapy, performing HCV-RNA and HBV DNA quantification every 2 months (III-A) for 1 year.

- Peg-IFN+Ribavirin, at standard doses and duration, is the therapy of choice in case of HCV replication (II-A).

- HBV reactivation may occur during Peg-IFN+Ribavirin therapy. Thus, HBV DNA levels must be monitored every 3 months during treatment.

- Treatment with anti-HBV analogs must be considered in case of constant or fluctuating HBV replication (HBV DNA $>2000 \mathrm{IU} / \mathrm{mL}$, presence of anti-HBc IgM), during the pre-treatment time or in case of reactivation during Peg-IFN+RBV therapy (IIIB). The choice of the analog must take into account the toxicity data in case of concomitant therapy with PegIFN+RBV (IV-A). Telbivudine must not be administered with Peg-IFN.

- Therapy with Peg-IFN alone can be continued in patients with initially active replication of both the viruses in case of non-response to HCV after 12 weeks of therapy (HCV-RNA decrease $<2 \mathrm{log}$ ), but with good response to HBV (IV-B)

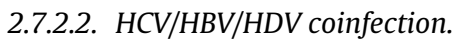

1. Patients with HCV, HBV, HDV co-infection should be monitored repeatedly by detecting HCV-RNA, HBV-DNA, anti HDV IgM and HDV-RNA to identify whom and how to treat (III-A).

2. Virological evaluation is recommended every 2 months during a 1-year period of observation (III-B).

3. Treatment is indicated in patients with persistent viremia, ALT $>$ ULN and liver histology showing significant fibrosis, at least S2 by Ishak Classification (IV-A).

4. Treatment with Peg-Interferon+RBV is suggested in patients with active $\mathrm{HCV}(\mathrm{V}-\mathrm{A})$. If an early virological response of at least $2 \log 10$ reduction in HCV-RNA compared to baseline is not achieved at week 12, RBV could be discontinued and PegInterferon monotherapy administered for 48-72 weeks. The duration of Peg-Interferon therapy should be defined also evaluating HDV replication (II-A).

5. During Peg-IFN/RBV therapy cirrhotic patients are recommended for a frequent check due to the side effects and the need of dose adjustment (I-A).

6. Treatment with Nucleos(t)ide analogues should be considered in patients with triple hepatic infection (B, C, D, ) and active HBV replication (HBV-DNA $>2000 \mathrm{UI} / \mathrm{mL}$ and anti-HBcIgM positive) (V-B).

\subsection{Treatment of HCV infection in elderly patients (Group coordinated by Giorgio Francesco Antonucci)}

\subsubsection{Background}

In Italy the epidemiology of HCV infection is characterised by increasing prevalence with age. The identification of elderly patients with chronic HCV infection is very common in clinical practice, and the decision to treat or not these patients is often difficult. In fact, the majority of multicenter registration trials excluded patients aged $>65$ years, thus making it difficult to know whether the efficacy of HCV treatments in such patients is any different.

Overall, the evidence maturated in subsequent studies indicate that virological response (SVR) rates tend to be lower in the elderly, and predictors of response are similar to those observed in younger individuals.

Previous AISF Guidelines (2003) stated that elderly HCVinfected patients may be treated on a case-by-case basis taking into account the severity of their liver disease and the presence of comorbidities, and that treatment should be limited to subjects with a life expectancy of $>5$ years.

\subsubsection{Statements}

1. HCV-infected subjects aged 65 years or more should be considered elderly patients (AIII).

2. Treatment is indicated in elderly patients, who are at increased risk of developing severe liver disease (BIV).

3. Peg-IFN plus weight-based ribavirin is the SOC for elderly patients with chronic hepatitis $C$; schedules and doses are the same as those recommended for younger patients (B-III).

4. Treatment decisions In elderly patients should be individualised on the basis of the severity of liver disease, HCV genotype, the presence of co-morbidities (particularly those related to aging), and the likelihood of severe side effects. Elderly patients with little or no liver fibrosis, and those with a life expectancy of less than 5 years should not be treated (B-III).

5. When possible, co-morbidities should be controlled by appropriate treatment before starting HCV therapy (A-III).

6 . The predictors of response and the criteria used to assess treatment success are the same in elderly and younger patients (B-III). 
2.9. Treatment of patients with normal ALT levels (Group coordinated by Claudio Puoti)

\subsubsection{Background}

The upper normal alanine aminotransferase (ALT) limit varies between studies and between tests performed in different laboratories because of technical reasons and different reference populations. Furthermore, the ALT reference ranges currently used in clinical practice underestimate the actual frequency of liver disease in this subset of patients as current evidence suggests that existing 'normal' ALT thresholds are too high and should be lowered by $25-30 \%$, thus setting the "optimal" ALT threshold at $30 \mathrm{U} / \mathrm{L}$ for males and $20 \mathrm{U} / \mathrm{L}$ for females. Finally, given the typically fluctuating pattern of ALT levels in chronic HCV infection, only more stringent tests can distinguish subjects with persistently normal ALT values (PNALT) from those in temporary biochemical remission. The definition of PNALT should be based on at least nine ALT determinations carried out every 2 months over an 18-month observation period.

The earliest guidelines discouraged interferon (IFN) treatment in patients with PNALT except in clinical trials because most have low-grade liver fibrosis and are at low risk of disease progression, because of the cost and side effects of therapy, and because of the low response rates to IFN monotherapy $(<10-15 \%)$ with a risk of ALT flares in up to $50 \%$ of patients during treatment. On the other hand, it has been stressed that these patients often have features traditionally associated with a good therapeutic response, such as mild histological lesions, and there is a prevalence of females and genotype non-1 infection. Finally, given the possibility of ALT flares during follow-up (which invariably accelerate the progression of fibrosis and the worsening of histological activity), the opportunity of deferring therapy has been questioned because of the possible risk of disease worsening.

The introduction of the new combination of pegylated IFN (PegIFN) plus ribavirin (RBV) has led to overall response rates of more than $50 \%$, and a favourable risk/benefit ratio even in patients with benign or slowly progressing disease. On this basis, an ad hoc AISF Committee and a more recent Medical Position Statement on the Management of Hepatitis $C$ by the American Gastroenterological Association (AGA) have noted that "decision analyses in patients with biochemically and histologically mild chronic hepatitis $C$ have led to the conclusion that, even in this population, antiviral therapy is cost-effective. Clinicians may rely in their decision making on individual patient features, including patient motivation and perspective, genotype, relative histological activity and fibrosis, duration of HCV infection, age, occupation, symptoms, and so on. Whether antiviral therapy is really cost-effective in HCV patients with PNALT has not yet been clearly proven. The possibility of even severe ALT flares among genotype 2 carriers, leading to progressive fibrosis and disease worsening, should be taken into account. In patients not receiving antiviral treatment, periodic measurements of ALT levels and adequate lifestyles should be recommended. In particular, overweight and the use of alcohol should be strongly discouraged.

\subsubsection{Statements}

1. Due to the fluctuating pattern of ALT levels in patients with chronic hepatitis $\mathrm{C}$, only more stringent tests will make it possible to distinguish subjects with persistently normal ALT values (PNALT) from those in transient biochemical remission. The definition of PNALT should be based on at least nine ALT determinations made at intervals of at least 2 months over an 18-month observation period. The optimal ALT threshold should be set at $30 \mathrm{U} / \mathrm{L}$ for males and $20 \mathrm{U} / \mathrm{L}$ for females (B-II).

2. HCV carriers with PNALT may receive antiviral treatment with Peg-IFN plus RBV using the same algorithms and protocols as those recommended for HCV patients with abnormal ALT levels (A-II). The Italian Medicines Agency restricts this prescription indication to Peg-IFN alpha2a.

3. Decision making should rely on individual characteristics such as genotype, histology, age, potential disease progression, the probability of viral eradication, patient motivation, the desire for pregnancy, comorbidities, co-factors, etc. (A-VI).

4. Antiviral treatment might be offered without the need for liver biopsy in patients with a high likelihood of achieving an SVR (e.g. an age of $<50$ years + easy-to-treat HCV genotype + low viral load), in the absence of any contraindication and co-factors of poor responsiveness $(\mathrm{A}-\mathrm{VI})$.

5. In patients aged 50-65 years, and in those with a reduced likelihood of achieving an SVR, a liver biopsy may be used to evaluate the need for therapy, with treatment being recommended only for patients with more severe fibrosis ( $>$ F2) and a higher possibility of response, depending on the HCV genotype (A-VI).

6. Biopsy and therapy are not recommended in therapy elderly $(>70$ years). These patients should be recommended to adopt lifestyle changes and undergo periodic ALT determinations (D-VI).

7. Non-invasive assessments of fibrosis can be used to detect changes over time and consequently indicate the need for biopsy or treatment on an individual patient basis.

8. In patients not receiving antiviral treatment, periodic ALT measurements and adequate lifestyles should be recommended. In particular, overweight and the use of alcohol should be strongly discouraged.

\section{Conflict of interest statement}

None declared.

\section{Acknowledgements}

Organizers: Daniele Prati (AISF Secretary), Antonio Gasbarrini (AISF Past Secretary), Francesco Mazzotta (SIMIT President), Evangelista Sagnelli (SIMIT), Giampiero Carosi (SIMAST President).

Expert Panel: Nicola Abrescia, Alfredo Alberti, Silvia Ambu, Piero Andreone, Angelo Andriulli, Mario Angelico, Giorgio Antonucci, Antonio Ascione, Luca Saverio Belli, Raffaele Bruno, Savino Bruno, Patrizia Burra, Calogero Cammà, Nicola Caporaso, Giuseppe Cariti, Umberto Cillo, Nicola Coppola, Antonio Craxì, Andrea De Luca, Eleonora De Martin, Vito Di Marco, Stefano Fagiuoli, Carlo Ferrari, Giovanni Battista Gaeta, Massimo Galli, Antonio Grieco, Paolo Grossi, Anna Licata, Ivana Maida, Alessandra Mangia, Nicoletta Marino, Renato Maserati, Gabriele Missale, Mario Mondelli, Paola Nasta, Grazia Niro, Marcello Persico, Enzo Petrelli, Antonio Picciotto, Fabio Piscaglia, Teresa Pollicino, Daniele Prati, Claudio Puoti, Massimo Puoti, Giovanni Raimondo, Maria Grazia Rumi, Evangelista Sagnelli, Teresa Santantonio, Antonina Smedile, Giovanni Squadrito, Gianluca Svegliati Baroni, Gloria Taliani, Marcello Tavio, Mario Toti.

Reviewers: Ferruccio Bonino, Maurizia Rossana Brunetto, Bruno Cacopardo, Marcello Caremani, Roberto Cauda, Massimo Colombo, Giovanni Di Perri, Francesca Donato, Patrizia Farci, Giovanna Fattovich, Gaetano Filice, Florio Ghinelli, Vincenzo Guadagnino, Adriano Lazzarin, Massimo Levrero, Giuseppe Licata, Anna Orani, Amerigo Paffetti, Giuseppe Pastore, Felice Piccinino, Eligio Pizzigallo, Patrizia Pontisso, Vincenzo Portelli, Mario Rizzetto, Alessandro Rossi, Tommaso Stroffolini, Enzo Ubaldi

Writing committee: Daniele Prati, Antonio Gasbarrini, Teresa Santantanio, Alfredo Alberti, Maria Grazia Rumi, Savino Bruno, Stefano Fagiuoli, Paolo Grossi, Raffaele Bruno, Giovanni Battista Gaeta, Giorgio Francesco Antonucci, Claudio Puoti, Calogero Cammà, Antonio Craxì. 
The writing committee is indebted with the A.I.S.F. secretariat for the helpful contribution during the preparation of the manuscript.

\section{Further reading}

\section{Treatment of acute hepatitis $C$}

[1] Alberti A, Bonino F, Bortolotti F, et al. Trattamento della epatite da HCV. Linee guida della Associazione Italiana per lo Studio del Fegato (AISF); 2004

[2] Licata A, Di Bona D, Schepis F, et al. When and how to treat acute hepatitis C? J Hepatol 2003;39:1056-62.

[3] Santantonio T, Fasano M, Sinisi E, et al. Efficacy of a 24-week course of PEGInterferon $\mathrm{a}-2 \mathrm{~b}$ monotherapy in patients with acute hepatitis $\mathrm{C}$ after failure of spontaneous clearance. J Hepatol 2005;42:329-33.

[4] Santantonio T, Medda E, Ferrari C, et al. Risk factors and outcome among a large patient cohort with community-acquired acute hepatitis $\mathrm{C}$ in Italy. Clin Infect Dis 2006;43(9):1154-9.

[5] Santantonio T, Sinisi E, Guastadisegni A, et al. Natural course of Acute Hepatitis C: a long-term prospective study. Dig Liver Dis 2003;35:104-13.

[6] Santantonio T, Wiegand J, Tilman Gerlach J. Acute hepatitis C: Current status and remaining challenges. J Hepatol 2008;49:625-33.

[7] Strader DB, Wright T, Thomas DL, et al. Diagnosis, management, and treatment of hepatitis C. Hepatology 2004;39(4):1147-71.

\section{Individualised therapy for chronic hepatitis $C$}

[8] Berg T, Von wagner M, Nasser S, et al. Extended treatment duration for hepatitis C virus type 1: comparing 48 versus 72 weeks of Peginterferon-alfa-2a plus ribavirin. Gastroenterology 2006;130:1086-97.

[9] Dalgard O, Bjøro K, Hellum KB, et al. Treatment with pegylated interferon and ribavarin in HCV infection with genotype 2 or 3 for 14 weeks: a pilot study. Hepatology 2004;40:1260-5.

[10] Dalgard O, Bjøro K, Ring-Larsen $\mathrm{H}$, et al. Pegylated interferon alfa and ribavirin for 14 versus 24 weeks in patients with hepatitis $C$ virus genotype 2 or 3 and rapid virological response. Hepatology 2008:47(1):35-42.

[11] Ferenci P, Berghoiz U, Laferi $H$, et al. 24 week treatment regimen with peginterferon alfa-2a (40 kd)plus ribavirin in HCV genotype 1 or 4 "super-responders" EASL abstract. J Hepatology 2006;44(Suppl. 2):8A.

[12] Ferenci P, Laferl H, Scherzer TM, et al. Peginterferon alpha-2a and ribavirin for 48 weeks in hepatitis C type 1 and 4 ptients with rapid virological response. Gastroentrology 2008;135:451-8.

[13] Jensen D, Morgan TR, Marcellin P, et al. Early identification of HCV genotype 1 patients responding to 24 weeks Peginterferon alfa-2a ribavirin therapy. Hepatology 2006;43:954-60.

[14] Mangia A, Santoro R, Minerva N, et al. Peginterferon alfa-2b and ribavirin for 12 vs. 24 weeks in HCV genotype 2 or 3. N Engl J Med 2005;352(25):2609-17.

[15] Mangia A, Minerva N, Bacca D, et al. Individualized treatment duration for hepatits C genotype 1 patients: a randomized controlled trial. Hepatology 2008:47:43-50.

[16] Sanchez-Tapias JM, Diago M, Escartìn P, et al. Peginterferon-alfa2a plus ribavirin for 48 versus 72 weeks in patients with detectable hepatitis $C$ virus RNA at week 4 of treatment. Gastroenterology 2006;131:451-60.

[17] Shiffman ML, Suter F, Bacon BR, et al. Peginterferon alfa-2a and ribavirin for 16 or 24 weeks in HCV genotype 2 or 3. N Engl J Med 2007;357(2):124-34.

[18] von Wagner M, Huber M, Berg T, et al. Peginterferon-alpha-2a (40KD) and ribavirin for 16 or 24 weeks in patients with genotype 2 or 3 chronic hepatitis $C$. Gastroenterology 2005;129(2):522-7.

[19] Zeuzem S, Buti M, Ferenci P, et al. Efficacy of 24 weeks treatment with Peginterferon alfa-2b plus ribavirin in patients with chronic hepatitis $C$ infected with genotype 1 and low pretreatment viremia. J Hepatol 2006:1.

\section{Retreatment of non-responders and relapsers}

[20] Ciancio A, Picciotto A, Giordanino C, et al. A randomized trial of pegylatedinterferon-alpha2a plus ribavirin with or without amantadine in the re-treatment of patients with chronic hepatitis $C$ not responding to standard interferon and ribavirin. Aliment Pharmacol Ther 2006;24(October (7)):1079-86

[21] Diago M, Crespo J, Olveira A, et al. Clinical trial: pharmacodynamics and pharmacokinetics of re-treatment with fixed-dose induction of peginterferon alpha-2a in hepatitis $\mathrm{C}$ virus genotype 1 true non-responder patients. Aliment Pharmacol Ther 2007:26(October (8)):1131-8.

[22] Jacobson IM, Gonzalez SA, Ahmed F, et al. A randomized trial of pegylated interferon alpha-2b plus ribavirin in the retreatment of chronic hepatitis C. Am J Gastroenterol 2005;100(11):2453-62.

[23] Jensen DM, Marcellin P, Freilich B, et al. Re-treatment of chronic hepatitis C non-responders to peginterferon alfa-2b: a randomized trial. Ann Intern Med 2009;150(April (8)):528-40.

[24] Krawitt EL, Ashikaga T, Gordon SR, et al. Peginterferon alfa-2b and ribavirin for treatment-refractory chronic hepatitis C. J Hepatol 2005;43(2):243-9.

[25] Maynard M, Pradat P, Bailly F, et al. Amantadine triple therapy for nonresponder hepatitis $\mathrm{C}$ patients. Clues for controversies (ANRS HC 03 BITRI). J Hepatol 2006;44(3):484-90.

[26] Moucari R, Ripault MP, Oulès V, et al. High predictive value of early viral kinetics in retreatment with peginterferon and ribavirin of chronic hepati- tis $C$ patients non-responders to standard combination therapy. J Hepatol 2007;46(4):596-604.

[27] Poynard T, Colombo M, Bruix J, et al. Peginterferon alfa-2b and Ribavirin: Effective in Patients With Hepatitis C Who Failed Interferon-alfa/Ribavirin Therapy. Gastroenterology 2009;136(5), 1618-28.32.

[28] Sherman M, Yoshida EM, Deschenes M, et al. Peginterferon alfa-2a (40KD) plus ribavirin in chronic hepatitis $\mathrm{C}$ patients who failed previous interferon therapy. Gut 2006;55(11):1631-8.

[29] Taliani G, Gemignani G, Ferrari C, et al. Pegylated interferon alfa-2b plus ribavirin in the retreatment of interferon-ribavirin nonresponder patients. Gastroenterology 2006;130(4):1098-106.

\section{Antiviral treatment in HCV cirrhosis}

[30] Abergel A, Hezode C, Leroy V, et al. Peginterferon-alfa2b plus ribavirin for treatment of chronic hepatitis $\mathrm{C}$ with severe fibrosis; a multicentre randomized trial comparing two doses of peginterferon-alfa2b. J Viral Hepat 2006;13:811-20.

[31] Bruno S, Maisonneuve P, Pockros PJ, et al. Efficacy and safety of peg-interferon alfa-2a (40 Kd) plus ribavirin in HCV patients with complete cirrhosis (Knodell score F4). Hepatology 2006;44(4 S1):A332-3.

[32] Bruno S, Stroffolini T, Colombo M, et al. Sustained virological response to interferon-alpha is associated with improved outcome in HCV-related cirrhosis: a retrospective study. Hepatology 2007;45:579-87.

[33] Di Bisceglie AM, Shiffman ML, Everson GT, et al. Prolonged therapy of advanced chronic hepatitis C with low-dose peginterferon. NEJM 2008;359:2429-41.

[34] Di Marco V, Almasio PL, Ferraro D, et al. Peg-interferon alone or combined with ribavirin in HCV cirrhosis with portal hypertension: a randomized controlled trial. J Hepatol 2007; 47:484-91.

[35] Everson GT, Hoefs JC, Seeff LB, et al. Impact of disease severity on outcome of antiviral therapy for chronic hepatitis C: Lessons from the HALT-C trial. Hepatology 2006;44:1675-84.

[36] Iacobellis A, Siciliano M, Perri F, et al. Peginterferon alfa-2b and ribavirin in patients with hepatitis $\mathrm{C}$ virus and decompensated cirrhosis: a controlled study. J Hepatol 2007;46(2):206-12.

[37] Marrache F, Consigny Y, Ripault MP, et al. Safety and efficacy of Peginterferon plus ribavirin in patients with chronic hepatitis $\mathrm{C}$ and bridging fibrosis or cirrhosis. J Viral Hepat 2005;12:421-8.

[38] Roffi L, Colloredo G, Pioltelli P, et al. Pegylated interferon-alpha2b plus ribavirin an efficacious and welltolerated treatment regimen for patients with hepatitis C virus related histologically proven cirrhosis. Antivir Ther 2008;13:663-73.

\section{Treatment of HCV reinfection after liver transplantation}

[39] Arjal RR, Burton Jr JR, Villamil F, Rosen HR. Review article: The treatment of hepatitis $\mathrm{C}$ virus recurrence after liver transplantation. Alimentary Pharmacol Ther 2007;26(2):127-40.

[40] Berenguer M, Palau A, Aguilera V, et al. Clinical benefits of antiviral therapy in patients with recurrent hepatitis C following liver transplantation. Am J Transplant 2008;8(3):679-87.

[41] Bizollon T, Pradat P, Mabrut JY, et al. Histological benefit of retreatment by pegylated interferon alfa-2b and ribavirin in patients with recurrent hepatitis C virus infection posttransplantation. Am J Transplant 2007;7(2):448-53.

[42] Carrión JA, Navasa M, García-Retortillo M, et al. Efficacy of antiviral therapy on hepatitis $C$ recurrence after liver transplantation: a randomized controlled study. Gastroenterology 2007:132(5):1746-56.

[43] Chalasani N, Manzarbeitia C, Ferenci P, et al. Peginterferon alpha-2a for hepatitis $\mathrm{C}$ after liver transplantation: two randomized, controlled trials. Hepatology 2005;41(Febuary (2)):289-98 [Erratum in: Hepatology 2005; 42(2):506].

[44] Charlton M. Approach to recurrent hepatitis C following liver transplantation. Current Gastroenterology Reports 2007;9(1):23-30.

[45] Cicinnati VR, Iacob S, Klein CG, et al. Ribavirin with either standard or pegylated interferon to treat recurrent hepatitis C after liver transplantation. Aliment Pharmacol Ther 2007;26(2):291-303.

[46] Hanouneh IA, Miller C, Aucejo F, et al. Recurrent hepatitis $C$ after liver transplantation: on-treatment prediction of response to peginterferon/ribavirin therapy. Liver Transpl 2008;14(1):53-8.

[47] Shiffman M. Treating chronic hepatitis C virus after liver transplantation: balancing the risks against the chance for success. Liver Transp 2007;13(8):1088-91.

\section{Treatment of HIV/HCV co-infection}

[48] Alberti A, Clumeck N, Collins S, et al. Short statement of the first European Consensus Conference on the treatment of chronic hepatitis B and C in HIVcoinfected patients. J Hepatol 2005;42:615-24.

[49] Mauss S, Valenti W, De Pamphilis J, et al. Risk factors for hepatic decompensation in patients with HIV/HCV coinfection and liver cirrhosis during interferon-based therapy. AIDS 2004;18:F21-5.

[50] Nasta P, Gatti F, Puoti M. Insulin resistance impairs rapid virologic response in HIV/hepatitis C virus coinfected patients on peginterferon-alfa-2a. AIDS 2008 Apr 23;22(7):857-61.

[51] Nunez M, Miralles C, Berdon MA, et al. The PRESCO study group: role of weightbased ribavirin dosing and extended duration of therapy in chronic hepatitis C in HIV-infected patients. The PRESCO trial. AIDS Res Human Retroviruses 2007;23:972-82.

[52] Opravil M, Sasadeusz J, Cooper DA, et al. Effect of baseline CD4 cell count on the efficacy and safety of peginterferon alfa-2 $(40 \mathrm{KD})$ plus ribavirin in 
patients with HIV-HCV co-infection. J Acquir Immune Def Syndr 2008;47: 36-49.

[53] Rockstroh JK, Bhagani S, Benhamou Y, Bruno R. EACS Executive Committee European AIDS Clinical Society (EACS) guidelines for the clinical management and treatment of chronic hepatitis B and C coinfection in HIV-infected adults. HIV Medicine 2008;9:82-8.

[54] Romero-Gómez M, Del Mar Viloria M, Andrade RJ. Insulin resistance impairs sustained response rate to peginterferon plus ribavirin in chronic hepatitis $C$ patients. Gastroenterology 2005;128(3):636-41.

[55] Torriani FJ, Rodriguez-Torris M, Rockstroh JK, et al. Peginterferon alfa-2a plus ribavirin for chronic hepatitis $\mathrm{C}$ virus infection in HIV infected patients. N Engl J Med 2004;351:438-50.

Treatment of $\mathrm{HCV} / \mathrm{HBV} \pm \mathrm{HDV}$ co-infection

[56] Farci P, Chessa C, Balestrieri C, et al. Treatment of chronic hepatitis D. Med Virol 2007;14:58-63.

[57] Gaeta GB, Precone DF, Cozzi-Lepri A, et al. Multiple viral infections. J Hepatol 2006;44:S108-13.

[58] Pontisso P, Ruvoletto MG, Fattovich G, et al. Clinical and virological profiles in patients with multiple hepatitis virus infections. Gastroenterology 1993;105:1529-33.

[59] Liaw YF. Role of hepatitis $C$ virus in dual and triple hepatitis virus infection. Hepatology 1995;22:1101-8.

[60] Gaeta GB, Stornaiuolo G, Precone DF, et al. Epidemiological and clinical burden of chronic hepatitis B virus/hepatitis $C$ virus infection. A multicenter Italian study. J Hepatol 2003;39:1036-41.

[61] Niro GA, Ciancio A, Gaeta GB, et al. Pegylated interferon alpha-2b as monotherapy or in combination with Ribavirin in Chronic Hepatitis Delta. Hepatology 2006;44:713-20.

[62] Raimondo G, Brunetto MR, Pontisso P, et al. Longitudinal evaluation reveals a complex spectrum of virological profiles in hepatitis $B$ virus/hepatitis $C$ viruscoinfected patients. Hepatology 2006;43:100-7.

[63] Carosi G, Rizzetto M. Treatment of chronic hepatitis B: Recommendations from an Italian workshop. Dig Liver Dis G 2008;40:603-17.

\section{Treatment of HCV infection in elderly patients}

[64] Alessi N, Freni MA, Spadaro A, et al. Efficacy of interferon treatment (IFN) in elderly patients with chronic hepatitis C. Infez Med 2003;11(4):208-12.

[65] Andreone P, et al. Infezione da HCV in età geriatrica, fase retrospettiva. In: AISF Annual Meeting. Oral Presentation. 2008.
[66] Antonucci G, Longo MA, Angeletti $C$, et al. The effect of age on response to therapy with peginterferon alpha plus ribavirin in a cohort of patients with chronic HCV hepatitis including subjects older than $65 \mathrm{yr}$. Am J Gastroentero 2007;102(7):1383-91.

[67] Bacosi M, Russo F, D'innocenzo S, et al. Amantadine and interferon in the combined treatment of hepatitis $C$ virus in elderly patients. Hepatol Res 2002;22(3):231-9.

[68] Iwasaki Y, Ikeda H, Araki Y, et al. Limitation of combination therapy of interferon and ribavirin for older patients with chronic hepatitis C. Hepatology 2006;43(1):54-63.

[69] Kumada T, Toyoda $H$, Honda $T$, et al. Treatment of chronic hepatitis $C$ with interferon alone or combined with ribavirin in Japan. Intervirology 2006;49: 112-8.

[70] Thabut D, Le Calvez S, Thibault V, et al. Hepatitis C in 6, 865 patients 65 yr or older: a severe and neglected curable disease? Am J Gastroenterol 2006;101(6):1260-7

Treatment of patients with normal ALT levels

[71] Bacon BR. Treatment of patients with Hepatitis $C$ and normal serum aminotransferase levels. Proc of the NIH Consensus Conference Management of Hepatitis C Hepatology 2002;36(Suppl. 1):S179-84.

[72] Prati D, Taioli E, Zanella A, et al. Updated definitions of healthy ranges for serum alanine aminotransferase levels. Ann Intern Med 2002;137:1-10.

[73] Puoti C, Guido M, Mangia A, et al. Clinical management of HCV carriers with normal aminotransferase levels. Dig Liver Dis 2003;35:362-9 [2006 update on http://www.webaisf.org/commconl.htm].

[74] Puoti C, Pellicelli AM, Romano M, et al. on behalf of the Hospital Liver Club. Antiviral treatment of HCV carriers with normal ALT. 43rd Meeting EASL, poster \# 826, J Hepatol 2008; 48: S309-310.

[75] Snoeck E, Hadziyannis SJ, Puoti C, et al. Predicting efficacy and safety outcome in patients with hepatitis $C$ virus genotype 1 and persistently 'normal' alanine aminotransferase levels treated with peginterferona-2a (40 KD) plus ribavirin. Liver Int 2008;28:61-71.

[76] Strader DB, Wright T, Thomas DL, Seef LB. Diagnosis, management and treatment of hepatitis C. AASLD Practice Guideline. Hepatology 2004;39: 1147-71

[77] Zeuzem S, Diago M, Gane E, et al. Peginterferon alfa-2a [40 KD] and ribavirin in patients with chronic hepatitis $\mathrm{C}$ and normal aminotransferase levels. Gastroenterology 2004;127:1724-32. 